\title{
The New Markers of Ventricular Dispersion in Patients with Acute Poisoning with TCAs: Tp-e Interval and Tp-e/QTc Ratio
}

\author{
Akkan Avci ${ }^{1, *}$, Mehmet Uzucek ${ }^{1}$, Satuk BugraYapici ${ }^{1}$, Begum Seyda Avci², \\ Onder Yesiloglu ${ }^{1}$, Hayri Cinar ${ }^{1}$, Ferhat Icme ${ }^{3}$, Salim Satar ${ }^{1}$
}

\author{
${ }^{1}$ Department of Emergency Medicine \\ Adana City Research and Training \\ Hospital, Health Science University, \\ Adana, Turkey \\ ${ }^{2}$ Department of Internal Medicine, \\ Adana City Research and Training \\ Hospital, Health Science University, \\ Adana, Turkey \\ ${ }^{3}$ Department of Emergency Medicine, \\ Ankara City Hospital, Ankara, Turkey
}

\section{*Correspondence}

drakkanavci@gmail.com

(Akkan Avci)

\begin{abstract}
Introduction: Purpose of this study was to investigate the changes in Tp-e/QTc rates and Tp-e intervals in patients poisoned with tricyclic antidepressants. Methods: 187 patients who were poisoned with tricyclic antidepressants and admitted to our emergency department were included in this study. Control group consisted of 187 healthy individuals with similar age and gender characteristics. All patients underwent 12-lead electrocardiograms (ECG). In addition to QTc measurement, Tp-e/QTc ratios and Tp-e interval were measured in the ECG. Study data were divided two groups as patients and healthy controls. Results: It was found that the QTc interval, Tp-e interval, and Tp$\mathrm{e} / \mathrm{QTc}$ ratio were significantly higher in the patient group compared to control group. The analysis demonstrated that QTc interval; Tp-e interval and Tp-e/QTc ratio were independently associated with heart rate. Conclusion: In poisoning with TCAs, the rate of QTc interval, Tp-e/QTc and Tp-e interval has increased and is independently associated with the heart rate in these patients. This may be the precursor of possible fatal ventricular arrhythmias in tricyclic antidepressants.
\end{abstract}

\section{Keywords}

Tricyclic antidepressants, QTc interval, Tp-e/QTc ratio Tp-e interval, Emergency medicine

\section{Introduction}

Acute poisoning with tricyclic antidepressants (TCAs) is one of the common causes of poisoning presenting to the emergency room. Electrocardiographic (ECG) changes are the most important marker detecting severe poisoning with anticholinergic agents such as the TCAs. The presence of abnormal ECG findings is a precursor of serious complications. Findings seen on electrocardiography are prolongation of the PR interval, widening of the QRS complex, long QT interval, AV block, tachycardia and an increase in $\mathrm{R} / \mathrm{S}$ ratio in the aVR derivation. Careful evaluation is important as rhythm disorders may develop that will increase mortality $[1,2]$.

ECG changes can be a precursor to ventricular arrhythmias. These changes include the distance from the peak of $\mathrm{T}$ wave to its end (Tp-e interval), QT and QTc time and QT and QTc dispersion. Ventricular transmural dispersion during repolarization is associated mostly with $\mathrm{Tp}$-e/QTc ratios [3] while Tp-e is usually a sign of ventricular repolarization. A prolonged Tp-e interval increases the risk of ventricular arrhythmias and abnormal repolarization in the ventricles [4]. To our knowledge, there is no study on Tp-e interval and Tp$\mathrm{e} / \mathrm{QTc}$ ratio used in the ventricular repolarization evaluation in acute poisoning with TCAs in recent years.
Purpose of this study is to observe changes in Tp-e/QTc ratios and Tp-e interval associated with TCA poisoning.

\section{Method}

\subsection{Study Design}

This was a retrospective study of patient records. The clinical records of patients admitted to Adana City Training and Research Hospital emergency medicine clinic due to poisoning between 01 January 2018 and 31 December 2019 were examined. A total of 1985 records were examined, and 283 patient records with features of TCA toxicity were observed. Of the 283 patient records reviewed, 69 patients were taking multiple drugs and/or taking active substances other than TCA and were excluded from the study. Exclusion criteria for the remaining 214 patients whose files have been reviewed are as follows: pharmacological treatments which would lengthen or shorten the QTc range, a family history of sudden cardiac arrest or syncope in the relations and patients, systemic or local acute or chronic infection, being under 18 years of age, failure to measure Tp-e and QTc in the ECG analysis, coronary artery disease (CAD) history or major CAD risk factors, diabetes mellitus, hypertension, medium-advanced valvular valve disease, systolic and diastolic heart failure and lack of file data. 


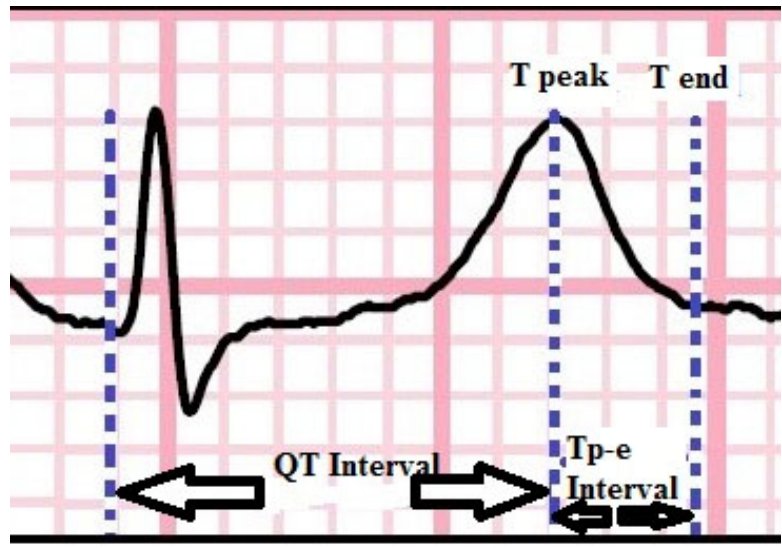

F I G URE 1. Measurement of QT and Tp-e Interval on electrocardiograpy.

A final total of 187 patients met the inclusion criteria and were included in the analysis. A comparative group of 187 healthy patients with sex and age characteristics close to the patient group were included as a control group. The study was conducted with the approval of the Local Ethics Committee (Adana City Education and Research Hospital, Clinical Research Ethics Committee, Date: 27.02.2020, Meeting Number: 51, Decision Number: 729), and all procedures followed were in accordance with the ethical standards of Helsinki Principles.

Demographic information and vital signs of both patient and control groups were scanned and age and sex characteristics, heart rates, blood pressure values of all patients and controls were recorded in the data registry form. Kidney function tests, sodium, potassium, calcium levels and liver function test parameters were measured from obtained blood samples.

\subsection{Evaluation of ECG}

The surface 12-lead electrocardiograms (ECG), set with a paper speed of $25 \mathrm{~mm} / \mathrm{s}$ and $10 \mathrm{~mm} / 1 \mathrm{mV}$ calibration, were analysed (MAC 2000 ECG Machine, GE medical systems information technologies, Inc., WI, USA). Presence of the sinus rhythm on the ECG was accepted as the inclusion criteria. The QT time was calculated as the interval between the start of the $\mathrm{Q}$ wave and where $\mathrm{T}$ wave ends in the isoelectric line. (Fig. 1). QTc in patients with heart rate between 60 $100 /$ minute was accounted for by using the Bazett Formula $(\mathrm{QTc}=\mathrm{QT} / \sqrt{ } \mathrm{R}-\mathrm{R})$. Frederica Formula $(\mathrm{QTc}=\mathrm{QT} / \mathrm{RR} 1 / 3)$ was used in order to calculate the QTc of patients with heart rate outside the range of $60-100 /$ minute. Tp-e interval was defined as the time between the peak point of the $\mathrm{T}$ wave and the point $\mathrm{T}$ wave connects to the isoelectrical line (Fig. 1). V5 lead was used as the primary lead for all the measurements. In cases where V5 was not suitable (amplitude $<1.5 \mathrm{~mm}$ ), calculations were made from the leads V4 or V6 [8]. Tp-e/QTc ratio was calculated based on these measurements. All ECG recordings were evaluated by two cardiologists unaware of the patients clinical condition, who have experience of six years or more in electrophysiology and evaluate more than 2000 arrhythmia patients yearly.

\subsection{Statistical Analysis}

SPSS 22.0 (Chicago, IL, USA) statistical software package were used to perform all analyzes. Kolmogorov-Smirnov test was used to determine whether the distribution of continuous variables was normal. Mean \pm standard deviation was used to present continuous variables and categorical variables were presented as numbers and percentages. Continuous variables with normal distribution were compared using the Student $t$ test, whereas the Mann-Whitney U test was used to compare variables that do not follow a normal distribution. Categorical variables were compared using Chi-square $\left(\chi^{2}\right)$ test. We use kappa coefficient to examine all ECG measurements for interobserver variability. The presence of a relationship between countable parameters was determined using Pearson's and Spearman's correlation analysis. $\mathrm{p}<0.05$ was accepted as statistical significant.

\section{Results}

Electrocardiographic evaluations were successfully completed in all patients. The Cohen kappa value for intra-observer and interobserver variability for all ECG criteria was over $90 \%$.

A comparison of the demographic data of the historical and study groups, demonstrated no difference regarding age and sex. Although the systolic and diastolic blood pressure, blood urea nitrogen and potassium values of the clinical parameters differed significantly between the groups, all four parameters were within normal limits. When compared to the control group, study subjects with tricyclic antidepressant overdose showed longer QTc and Tp-e intervals associated with a higher Tp-e/QTc ratio. (Table 1).

QTc, Tp-e interval and Tp-e/QTc measurements were positively correlated with the heart rate (Table 2). In linear regression analysis, Tp-e-interval and $\mathrm{Tp}-\mathrm{e} / \mathrm{QTc}$ ratio were detected to be independently related with heart rate (Table 3).

In the Scatterplot analysis performed with the Tp-e interval, QTc interval and Tp-e/QTc ratios with heart rate, $\mathrm{R}^{2}$ linear values were determined as $0.266,0.311$ and 0.285 , respectively (Table 3).

\section{Discussion}

The main finding of our study is that Tp-e/QTc ratios, QTc interval and Tp-e intervals were significantly higher in the group of patients poisoned with TCAs than the control group. Literature research shows there are no other published studies investigating the changes in the Tp-e interval and Tp-e/QTc ratios in the patients with TCA overdose. Depolarization of the ventricular myocardium occurs before ventricular repolarization and takes place from the endocardial region to the epicardial region. During ventricular repolarization there is dispersion between the epicardial and endocardial region. The Tp-e interval, is associated with transmural ventricular repolarization, is the interval between the $\mathrm{T}$ wave peak and the end distance $[3,6]$. The Tp-e/QTc ratios and Tp-e interval predispose to a high risk of sudden cardiac death and are associated with arrhythmias in many pathological cardiac conditions [4, 7-9]. The dispersion of repolarization between endocardial and epicardial region in the ventricular myocardium causes 
T A B L E 1. Comparison of demographic and laboratory findings groups.

\begin{tabular}{|lccc|}
\hline & Patients Group $(\mathbf{n}=\mathbf{1 8 7})$ & Control Group $(\mathbf{n}=\mathbf{1 8 7})$ & $\mathbf{p}$ \\
\hline Age (years) & $29.91 \pm 8.12$ & $29.37 \pm 8.23$ & 0.528 \\
\hline Systolic blood pressure (mmHg) & $116.36 \pm 12.43$ & $107.59 \pm 13.60$ & $<0.001$ \\
\hline Diastolic blood pressure (mmHg) & $71.76 \pm 8.46$ & $70.00 \pm 7.90$ & 0.038 \\
\hline Heart rate (pulse/minute) & $\mathbf{9 9 . 3 3} \pm \mathbf{9 . 5 6}$ & $\mathbf{7 6 . 5 7} \pm \mathbf{9 . 8 0}$ & $<\mathbf{0 . 0 0 1}$ \\
\hline Urea (mg/dL) & $34.38 \pm 8.96$ & $37.00 \pm 7.02$ & $<0.001$ \\
\hline Creatinine (mg/dL) & $0.67 \pm 0.18$ & $0.70 \pm 0.19$ & 0.084 \\
\hline Sodium (mEq/L) & $138.84 \pm 2.12$ & $138.68 \pm 2.04$ & 0.471 \\
\hline Potassium (mEq/L) & $\mathbf{4 . 0 9} \pm \mathbf{0 . 4 0}$ & $\mathbf{4 . 2 0} \pm \mathbf{0 . 4 5}$ & $\mathbf{0 . 0 0 8}$ \\
\hline Glucose (mg/dL) & $94.63 \pm 10.27$ & $99.21 \pm 10.21$ & $<0.001$ \\
\hline ALT (u/L)* & $19.88 \pm 7.59$ & $20.83 \pm 6.96$ & 0.207 \\
\hline AST (u/L)** & $25.11 \pm 7.55$ & $23.71 \pm 6.82$ & 0.061 \\
\hline Calcium (mg/dL) & $9.48 \pm 0.26$ & $9.52 \pm 0.27$ & 0.114 \\
\hline QTc (msec) & $\mathbf{4 5 9 . 2 2} \pm \mathbf{2 7 . 7 2}$ & $\mathbf{4 1 1 . 0 2} \pm \mathbf{1 3 . 6 5}$ & $<\mathbf{0 . 0 0 1}$ \\
\hline Tp-e (msec) & $\mathbf{9 4 . 2 2} \pm \mathbf{1 4 . 7 6}$ & $\mathbf{6 4 . 5 1} \pm \mathbf{1 0 . 4 3}$ & $<\mathbf{0 . 0 0 1}$ \\
\hline Tp-e/QTc Ratio (\%) & $\mathbf{2 0 . 4 3} \pm \mathbf{2 . 4 5}$ & $\mathbf{1 5 . 6 8} \pm \mathbf{2 . 4 8}$ & $<\mathbf{0 . 0 0 1}$ \\
\hline
\end{tabular}

*ALT: Alanine aminotransferase, **AST: Aspartate aminotransferase.

TA B L E 2. Correlation of QTe, Tp-e-interval and Tp-e/QTe raito with heart rate.

\begin{tabular}{cccccccc} 
& \multicolumn{2}{c}{ QTe } & \multicolumn{2}{c}{ Tp-e-interval } & \multicolumn{2}{c}{ Tp-e/QTe Ratio } \\
& $\mathbf{r}$ & $\mathbf{p}$ & $\mathbf{r}$ & $\mathbf{p}$ & $\mathbf{r}$ & $\mathbf{p}$ \\
Heart rate (pulse/minute) & 0.534 & $<\mathbf{0 . 0 0 0}$ & 0.558 & $<\mathbf{0 . 0 0 0}$ & 0.516 & $<\mathbf{0 . 0 0 0}$
\end{tabular}

TA B L E 3. A linear regression analysis for heart rate significantly correlated with QTe, Tp-e interval and Tp-e/QTe ratio.

\begin{tabular}{|ccccccc} 
& \multicolumn{2}{c}{ QTc } & \multicolumn{2}{c}{ Tp-e-Interval } & \multicolumn{1}{c}{ Tp-e/QTe Ratio } \\
& $\boldsymbol{\beta}$ & $\mathbf{p}$ & $\boldsymbol{\beta}$ & $\mathbf{p}$ & $\boldsymbol{\beta}$ & $\mathbf{p}$ \\
Heart rate (pulse/minute) & 0.534 & $<\mathbf{0 . 0 0 1}$ & 0.558 & $<\mathbf{0 . 0 0 1}$ & 0.516 & $<\mathbf{0 . 0 0 1}$ \\
\hline
\end{tabular}

$R^{2}$ Linear for $Q T c, T p$-e interval and Tp-e/QTc ratio as $0.285,0.311,0.266$, respectively.

slow transmission to these two anatomic regions and is thought to increase re-entry-related arrhythmias.

The effects of TCAs on the heart are realized by voltagedependent $\mathrm{Na}^{+}$channel and $\mathrm{K}^{+}$channel blockade, and postsynaptic inhibition of central and peripheral $\alpha$-adrenergic receptors. Voltage-dependent $\mathrm{Na}^{+}$channel blockade is the root cause of cardiotoxicity (arrhythmias, conduction blocks and hypotension) caused by TCA's. Inhibition of fast $\mathrm{Na}^{+}$channels in His-Purkinje cells causes depolarization delay and conduction abnormality. $\mathrm{Na}^{+}$channel blockade results in elongation of the action potential in Phase 0 , tachycardia, hypotension, hyponatremia and acidosis. $\mathrm{Na}^{+}$channel blockade leads to hypotension, various heart blocks, right axis deviation, QRS enlargement and ectopic heart beats. In addition, through voltage-dependent $\mathrm{K}^{+}$channel blockade, bradycardia and prolonged QTc interval are observed [10]. The most common rhythm disorder of associated with TCA toxicity is sinus tachycardia. Non-uniform ventricular conduction due to anterograde conduction may also cause ventricular tachycardia. The prolongation of the QT interval is an ECG finding that can be seen with either therapeutic or toxic doses of TCAs. QT prolongation is due to slowed depolarization. Patients with QT prolongation are prone to torsades de pointes rhythm [11].
Our study demonstrates that prolongation of the QTc interval, and increases in the Tp-e interval and Tp-e/QTc ratio predict prolongation of ventricular prolongation.

There are many studies which show QTc increases in acute TCA toxicity [12-14]. Okayasu et al examined the effect of antidepressants on the change of QTc interval, Tp-e interval and $\mathrm{Tp}-\mathrm{e} / \mathrm{QTc}$ ratio and found that there was an increase in the QTc ratio, but not in the Tp-e interval and Tp-e/QTc ratio [15]. However, this research was conducted on patients using prescribed doses of TCAs. There is no study investigating Tpe interval and Tp-e/QTc ratio in TCA toxicity. Our research also demonstrates that increase in the heart rate of patients has a significant correlation with the increase in Tp-e interval and Tp-e/QTc ratio in addition to the QTc interval. This finding may be related to cardiac toxicity caused by competitive inhibition of $\mathrm{Na}^{+}$and $\mathrm{K}^{+}$channels of TCA's.

\section{Conclusion}

In addition to QTc evaluation of patients with acute poisoning with TCA during routine ECG evaluation, the measurement of Tp-e-interval and Tp-e/QTc ratios, should be made. An elevation of these values may be an early precursor of ventric- 
ular arrhythmias. Further studies are needed to support these findings.

\section{LIMITATION OF STUDY}

The retrospective design of the study is an important limitation of our study. Further research with prospective design and higher number of patients can provide more information about this subject.

\section{ACKNOWLEDGEMENTS}

The authors thank Prof. Dr. Koc Mevlut, MD, from University of Health Science, Adana City Research and Training Hospital, Department of Cardiology, Arrhythmia Clinic and Dr. Koca Hasan, MD, from University of Health Science, Adana City Research and Training Hospital, Department of Cardiology, Arrhythmia Clinic for their efforts in evaluation and measurements of ECGs. We also thank Ass. Prof. Dr. Sumbul Hilmi Erdem MD, from University of Health Science, Adana City Research and Training Hospital, Department of Internal Medicine, for help with the preparation of this manuscript. Thanks to all the peer reviewers and editors for their opinions and suggestions.

\section{CONFLICT OF INTEREST}

The authors have no conflicts of interests to declare.

\section{AUTHOR CONTRIBUTIONS}

Avci Akkan, Satar Salim, and Onder Yesiloglu conceived and planned the study. Avci Akkan and Onder Yesiloglu carried out the study. Uzucek Mehmet, Yapici Satuk Bugra and Cinar Hayri provided all data. Avci Akkan, Icme Ferhat and Satar Salim were involved in planning and supervised the work, Avci Begum Seyda and Cinar Hayri processed the study data, performed the analysis, and designed the figures. Avci Akkan, Uzucek Mehmet, Yapici Satuk Bugra, Avci Begum Seyda, Yesiloglu Onder, Cinar Hayri and Satar Salim contributed to the design and implementation of the research, to the analysis of the results and to the writing of the manuscript.

\section{ETHICAL APPROVAL}

The ethics committee of the Adana City Training and Research Hospital approved the study.

\section{INFORMED CONSENT}

Written informed consent was not necessary because no patient data has been included in the manuscript.

\section{FUNDING}

This research did not receive any specific grant from funding agencies in the public, commercial, or not-for-profit sectors.

\section{AVAILABILITY OF DATA AND MATERIALS}

Data and materials are reachable from hospital automation information systems.

\section{HUMAN RIGHTS}

This manuscript was carried out in accordance with the Declaration of Helsinki and Good Clinical Practice guidelines.

\section{REFERENCES}

[1] Sık G, Nisli K, Çıtak A. Serious Cardiac Arrhythmia in Amitriptyline Poisoning: Case Report. Pediatr Emerg Intens Care Med. 2014;1:39-42.

[2] Otal Y, Karatas AD, Baydın A, et al. Ölümcül Doz Amitriptilin Zehirlenmesi: Olgu Sunumu. Frrat Tip Dergisi 2009;14:160-162.

[3] Kongstad O, Xia Y, Liang Y, et al. Epicardial and endocardial dispersion of ventricular repolarization. A study of monophasic action potential mapping in healthy pigs. Scand Cardiovasc J. 2005;39:342-347.

[4] Porthan K, Viitasalo M, Toivonen L, et al. Predictive Value of Electrocardiographic T Wave Morphology Parameters and T-Wave Peak to T-Wave End Interval for Sudden Cardiac Death in the General Population Clinical Perspective. Circ Arrhythm Electrophysiol. 2013;6:690-696.

[5] Chua KC, Rusinaru C, Reinier K, et al. Tpeak-to-Tend interval corrected for heart rate: A more precise measure of increased sudden death risk? Heart rhythm. 2016;13:2181-2185.

[6] Xia Y, Liang Y, Kongstad O, et al. Tpeak-Tend interval as an index of global dispersion of ventricular repolarization: evaluations using monophasic action potential mapping of the epi- and endocardium in swine. J Interv Card Electrophysiol. 2005;14:79-87.

[7] Gupta P, Patel C, Patel H, et al. T(p-e)/QT ratio as an index of arrhythmogenesis. J Electrocardiol. 2008;41:567-74.

[8] Erikssen G, Liestol K, Gullestad L, et al. The terminal part of the QT interval ( $\mathrm{T}$ peak to $\mathrm{T}$ end): a predictor of mortality after acute myocardial infarction. Ann Noninvasive Electrocardiol. 2012;17:85-94.

[9] Smetana P, Schmidt A, Zabel M, et al. Assessment of repolarization heterogeneity for prediction of mortality in cardiovascular disease: peak to the end of the $\mathrm{T}$ wave interval and nondipolar repolarization components. J Electrocardiol. 2011;44:301-308.

[10] Acikalin A, Gulen M, Acehan S, et al. All Supraventricular Tachycardia are not Cardiogenic: Case Report. JAEMCR. 2011;2:39-42.

[11] Pentel P, Benowitz N. Tricyclic antidepressant poisoning: management of arrhythmias. Med Toxicol. 1986;1:101-121.

[12] Niemann JT, Bessen HA, Rothstein RJ, et al. Electrocardiographic criteria for tricyclic antidepressant cardiotoxicity. Am J Cardiol. 1986;57:11541159.

[13] Caravati EM, Bossart PJ. Demographic and electrocardiographic factors associated with severe tricyclic antidepressant toxicity. J Toxicol Clin Toxicol. 1991;29:31-43.

[14] Acikalin A, Satar S, Avci A, et al. QTc intervals in drug poisoning patients with tricyclic antidepressants and selective serotonin reuptake inhibitors. Am J Ther. 2010;17:30-33.

[15] Okayasu H, Ozeki Y, Fujii K, et al. Investigation of the Proarrhythmic Effects of Antidepressants according to QT Interval, QT Dispersion and T Wave Peak-to-End Interval in the Clinical Setting. Psychiatry Investig. 2019;16:159-166.

How to cite this article:Akkan Avci, Mehmet Uzucek, Satuk BugraYapici, Begum Seyda Avci, Onder Yesiloglu, Hayri Cinar, et al. The New Markers of Ventricular Dispersion in Patients with Acute Poisoning with TCAs: Tp-e Interval and Tp-e/QTc Ratio. Signa Vitae. 2021;17(1):124-127. doi:10.22514/sv.2020.16.0044. 